\title{
Mapeo ultrasonográfico preoperatorio para optimizar tiempo de maduración de fístulas arteriovenosas
}

\section{Preoperative ultrasonographic mapping to optimize arteriovenous fistula maturation time}

\author{
Misael Silva-González, Wenceslao F. Mijangos*, Verónica Carbajal-Robles, Enrique Santillán-Aguayo, \\ Sandra Olivares-Cruz y Miguel Á. Sierra-Juárez \\ Servicio de Angiología, Cirugía Vascular y Endovascular, Unidad 503, Hospital General de México "Dr. Eduardo Liceaga”, Ciudad de México, \\ México
}

\begin{abstract}
Resumen
Antecedentes: La fístula arteriovenosa autóloga (FAV) es la mejor modalidad de acceso vascular para hemodiálisis por su permeabilidad primaria a largo plazo. Objetivo: Evaluar el mapeo ultrasonográfico preoperatorio y determinar si realizar este ayuda a disminuir el tiempo de maduración, comparado con aquellos a los que no se les realiza. Material y métodos: Se efectúo un estudio retrospectivo, descriptivo y analítico de los pacientes con fístula sin que se hiciera mapeo ultrasonográfico previo con aquellos pacientes que se sometieron a FAV realizando ultrasonido. Resultados: En total se analizaron 104 pacientes, de los cuales 51 correspondió al grupo de USG preoperatorio y 53 en el grupo clínico. No se observaron diferencias significativas entre ambos grupos. El grupo al que se le efectuó ultrasonido, maduraron 35 (33.7\%) y en el grupo con solo exploración física maduraron 35 (33.7\%). Sin embargo, se demostró que los pacientes a los que se les realizó ultrasonido previo maduraron antes que a los que no. Conclusiones: El ultrasonido preoperatorio demostró ser una herramienta indispensable, ya que permite realizar una mejor planeación y avance en los resultados de las fístulas.
\end{abstract}

Palabras clave: Fístula arteriovenosa. Ultrasonido. Evaluación preoperatoria.

\begin{abstract}
Background: The autologous arteriovenous fistula (AVF) is the best vascular access modality for hemodialysis due to its longterm primary patency. Objective: To evaluate the preoperative ultrasonographic mapping and determine if performing this assists to reduce the maturation time, compared to those without it. Material and methods: A retrospective, descriptive and analytical study of the patients with fistula was carried out without prior ultrasonographic mapping being done with those patients who underwent AVF using ultrasound. Results: A total of 104 patients were analyzed, of which 51 corresponded to the preoperative USG group and 53 to the clinical group. No significant differences were observed between both groups. The group that underwent ultrasound, $35(33.7 \%)$ matured and in the group with only physical examination, $35(33.7 \%)$ matured. However, it was shown that patients who underwent a previous ultrasound matured earlier than those who did not. Conclusions: Preoperative ultrasound demonstrated to be an indispensable tool, since it allows a better planning and progress in the results of fistulas.
\end{abstract}

Key words: Arteriovenous fistula. Doppler ultrasound. Preoperative evaluation.

Correspondencia:

*Wenceslao F. Mijangos

E-mail: cx_fabmw@ hotmail.com bajo la licencia CC BY-NC-ND license (http://creativecommons.org/licenses/by-nc-nd/4.0/).
Fecha de recepción: 12-02-2021

Fecha de aceptación: 06-05-2021

DOI: 10.24875/RMA.21000008
Disponible en internet: 24-09-2021

Rev Mex Angiol. 2021;49(3):74-79 www.RMAngiologia.com 


\section{Introducción}

La enfermedad renal crónica (ERC) surge como resultado de varias enfermedades heterogéneas que alteran la función y estructura del riñón de manera irreversible, durante meses o años ${ }^{1}$.

Se recomienda la creación de una fístula arteriovenosa (FAV) como abordaje inicial para el acceso a hemodiálisis $(H D)^{2}$. Se ha observado que si se inicia la HD por medio de una FAV existe una disminución en la mortalidad en comparación con los catéteres ${ }^{3}$. Sin embargo, la maduración de la FAV continua siendo un problema, con tasas de falla primaria que varían del 20 al $60 \% 4$.

Por lo tanto se deben enfocar los esfuerzos para lograr incrementar la proporción de FAV que logran una maduración adecuada para la diálisis debido a las altas tasas de fracaso que presentan ${ }^{5}$. Se propone que la exploración física (EP) es suficiente para aquellos pacientes que no presentan los principales factores de riesgo que se asocian con un fallo del acceso y las técnicas de imagen para evaluar la anatomía vascular (mapeo) complementaría a la EP para ayudar a predecir el éxito de la FAV, especialmente para aquellos pacientes de alto riesgo ${ }^{6}$. Sin embargo, la valoración preparatoria por USG da como resultado una tasa de fracaso menos inmediato, los pacientes presentan menos trombosis precoz y una mejor permeabilidad primaria asistida. El fracaso de la maduración sigue siendo un problema importante, incluso cuando se utiliza ecografía preoperatoria ${ }^{6}$.

En una revisión de Cochrane se concluye que no hay una evidencia clara que apoye el realizar un USG preoperatorio y que no hay evidencia estadísticamente significativa para realizarlo y que al contrario, puede dar lugar a citas adicionales, molestias al paciente y retrasar la cirugía. No se encontró que el USG preoperatorio reduzca el uso de los catéteres e incluso puede agregar costos innecesarios al sistema de salud. Sin embargo no se halló un consenso establecido de las técnicas para la obtención de imágenes de los vasos tanto arteriales como venosos y si la maduración en los pacientes sometidos a un USG preoperatorio maduraban antes ${ }^{8}$. Por estos antecedentes, la importancia de nuestro estudio radica en apoyar la evidencia actual de realizar ultrasonidos (USG) preoperatorios como parte de la evaluación integral de todo paciente candidato a construcción de FAV.

\section{Material y métodos}

Se realizó un estudio retrospectivo y analítico donde se revisó la base de datos del servicio de angiología de los pacientes sometidos a construcción de FAV de enero del 2013 a agosto del mismo año a los cuales se les hizo EP únicamente y se revisaron los expedientes de los pacientes sometidos a construcción de FAV a los cuales se les hizo mapeo ultrasonográfico preoperatorio del periodo de noviembre del 2018 a septiembre del 2019. Se realizó el cálculo del tamaño de muestra para análisis estadístico de diferencia de medias entre dos grupos independientes con un tamaño de efecto $d$ 0.5 , error alfa 0.5 y poder beta 0.8 , con lo que se requirió un tamaño de muestra de 51 pacientes en cada grupo ${ }^{5}$. Los criterios de inclusión fueron: pacientes con ERC en prediálisis o bajo tratamiento sustitutivo de la función renal y que tuviesen programada la realización de una FAV, edad $\geq 18$ años y $<60$ años, aquellos pacientes que aceptaron la realización del estudio y firmaron el consentimiento informado. Los criterios de exclusión fueron: pacientes con insuficiencia cardiaca grave (fracción de eyección del ventrículo izquierdo menor a 40\%), con hemoglobina menor de 7, albúmina sérica menor de $3.0 \mathrm{~g} / \mathrm{dl}$ e hipotensión ortostática previo al procedimiento quirúrgico, pacientes con estenosis de vasos centrales y pacientes con antecedente de trombofilia. Se realizó estadística descriptiva paramétrica y no paramétrica para la información epidemiológica y de los datos de las características clínicas de los pacientes a los que se les realizó FAV, se hicieron ajustes y transformaciones para obtener una distribución normal de los datos cuando fue necesario. Las variables cuantitativas con distribución normal se expresaron en media y desviación estándar, y cuando no se presentó distribución normal los datos se presentaron en mediana y rango intercuartil. Las variables cualitativas se expresaron en frecuencias absolutas y relativas. Se realizó cálculo de diferencia de medias de las variables de estudios de las características del procedimiento quirúrgico con prueba de $t$ de Student 0 prueba exacta de Fisher para las variables cualitativas, se calcularon diferencias de proporciones mediante prueba de chi cuadrada. Se realizó análisis del riesgo para alcanzar maduración mediante regresión logística de Cox. Se realizaron curvas de supervivencia para determinar el riesgo de maduración entre los grupos de estudio y se calculó la hazard ratio (HR) mediante regresión de Cox. Se estableció como significancia estadística un valor de $p<0.05$ bimarginal y un intervalo de confianza (IC) del 95\%. Este estudio no contempló la intervención o la manipulación del tratamiento de los pacientes del servicio de angiología y cirugía vascular a quienes se les realizó FAV. Únicamente contempló el análisis de los registros ya existentes. El 
estudio se llevó a cabo de conformidad con el Reglamento de la Ley General de Salud en Materia de Investigación en Salud e ICH-GCP (normas de buena práctica clínica, por sus siglas en inglés), con apego a la ética y salvaguarda de la privacidad y confidencialidad de la información obtenida, además de que la información se utilizará exclusivamente para fines académicos y de investigación.

\section{Resultados}

Se realizó un estudio retrospectivo, en donde se compararon pacientes a quienes se les hizo rastreo ultrasonográfico previo a la construcción de la FAV y a un grupo de pacientes a quienes no se les realizó mapeo preoperatorio en el Servicio de Angiología y Cirugía Vascular del Hospital General de México. En total se analizaron 104 pacientes, de los cuales 51 correspondieron al grupo con USG preoperatorio y 53 en el grupo clínico (GC), de los cuales alcanzaron maduración $33(33.7 \%)$ pacientes del GC y 35 (33.7\%) del grupo con USG con una $p=0.77$. Respecto a las características generales de la población, la edad promedio fue de $52 \pm 15.4$ años para el GC y de $47.6 \pm 14.4$ años para el grupo al que se le realizó USG, con una distribución de sexo masculino del GC 58.4 vs. $72.5 \%$ del grupo con USG, con una $p=0.13$; con respecto a las comorbilidades, no hubo diferencias significativas en cuanto diabéticos e hipertensos entre los grupos, la diálisis peritoneal como antecedentes se encontró en el GC en un $43.3 \%$ de pacientes y en un $17.6 \%$ en el grupo con USG preoperatorio con una $p=0.004$. La principal causa de enfermedad renal en ambos grupos fue diabetes, siendo en el GC 24 (45.2) y en el grupo de USG 27 (52.9); la segunda causa de enfermedad renal fue la causa indeterminada, con 18 (33.9) en el GC y de 12 (23.5) en el grupo de USG preoperatorio; la tercera causa fue hipoplasia renal en el grupo con USG 11 (21.5) y poliquistosis renal 6 (33.9) en el GC, con una $p=0.02$. El número de catéteres fue de $1.7 \pm$ 16.8 en el GC y de $2.08 \pm 1.67$ para el grupo con USG $(p=0.33)$ (Tabla 1).

Con respecto a las características quirúrgicas, los pacientes sometidos a fístulas radiocefálicas distales, en el grupo control fueron $8(15 \%)$ en el GC y $3(5.8 \%)$ en el grupo de USG; las braquicefálicas, 24 (45.2\%); braquimedianas, 10 (18.8\%); braquibasílicas, 5(9.8\%), y braquicomunicante posterior, 2 (3.7); en el GC, braquicefálicas, $25(49 \%)$; braquimedianas $13(25 \%)$, braquibasílica $5(9.8 \%)$ y braquicomunicante posterior $5(9.8 \%)$ en el grupo con USG, sin tener pacientes con fístulas braquiaxilares con injerto ni braquibraquiales con injerto de politetrafluroetileno (PTFE) en asa $(p=0.23)$. Con respecto al tipo de anastomosis, fueron término-lateral en 50 (94.3) y término-terminal 3 (5.6) en GC, y término-lateral 45 (88.2) y término-terminal 6 (11.7) en grupo USG. Los pacientes que presentaron thrill inmediato fueron 48 (90\%) en el GC y 46 (90.1\%) en el grupo USG, con una $p=0.94$. Presentaron maduración 35 (33.7\%) en el grupo cínico y 35 (33.7\%) en el grupo USG, con una $p=0.77$ (Tabla 2).

Se realizó un análisis multivariante para evaluar los factores de riesgo para alcanzar maduración, sin encontrar diferencias significativas en los grupos para sexo, edad, hipertensión, diabetes, causa de insuficiencia renal crónica (IRC), número de catéteres, antecedente de diálisis peritoneal, tiempo de HD, tiempo de IRC, tipo de fístula, tipo de anastomosis y thrill (Tabla 3).

Así mismo, se realizó un análisis multivariante con respecto a las características clínicas de los pacientes. Se tomaron en cuenta estas variables debido a que en otros artículos ya publicados dichos parámetros demostraron tener significancia estadística con respecto a la probabilidad de maduración ${ }^{4}$ (Tabla 4).

Se realizo el análisis multivariante con base en el tipo de fístula que se realizó y se encontraron diferencias estadísticamente significativas en las fístulas braquicefálicas ( $p=0.002$; HR: 0.27; 0.003-2.62), así como en las fístulas braquicomunicantes posteriores ( $p=0.003$; HR: $0.25 ; 0.002-0.285)$ con respecto a la maduración (Tabla 5).

Se realizó una curva de supervivencia con regresión de Cox para establecer diferencia entre el grupo al que se realizó USG y el grupo al que se le hizo solo EP para alcanzar maduración de la FAV, encontrando que en ambos grupos se alcanzó el mismo porcentaje de maduración, sin embargo, en el grupo al que se le hizo el rastreo ultrasonográfico alcanzó una maduración en menos tiempo de seguimiento, con una diferencia estadísticamente significativa (HR: 0.1; IC 95\%: 0.08-0.3; $p=0.001)$ (Fig. 1).

\section{Discusión}

La ERC con requerimiento sustitutivo de la función renal representa enormes costos mundiales. La FAV continúa siendo la mejor modalidad de acceso vascular para HD debido a que tiene la mejor permeabilidad primaria a largo plazo y requiere menos reintervenciones. El mapeo de los vasos sanguíneos utilizando técnicas de imagen previos a la construcción de una fístula 
Tabla 1. Características generales de la población

\begin{tabular}{|l|c|c|c|}
\hline Características & $\begin{array}{c}\text { Grupo clínico } \\
(\mathbf{n}=\mathbf{5 3})(\mathbf{1 0 0} \%)\end{array}$ & $\begin{array}{c}\text { Grupo ultrasonido } \\
(\mathbf{n}=\mathbf{5 1})(\mathbf{1 0 0} \%)\end{array}$ & $\mathbf{p}$ \\
\hline Edad (DE) & $52(98.1)$ & $47(92.1)$ & 0.36 \\
\hline Sexo (hombres) (\%) & $31(58.4)$ & $37(72.5)$ & 0.13 \\
\hline Hipertensión(\%) & $49(92.4)$ & $47(92.1)$ & 0.95 \\
\hline Diabetes (\%) & $30(56.6)$ & $29(56.8)$ & 0.97 \\
\hline Causa de enf. renal (\%) & & $27(52.9)$ & 0.02 \\
\hline Diabetes & $24(45.2)$ & $11(21.5)$ & 0 \\
\hline Hipoplasia renal \\
Enf. hipertensiva asociada al embarazo \\
IVU recurrente \\
Poliquistosis renal \\
Indeterminada & $1(1.6)$ & $1(1.9)$ & 0 \\
\hline Ant. diálisis peritoneal (\%) & $1(1.8)$ & $12(23.5)$ & 0.004 \\
\hline N. ${ }^{0}$ de catéteres (DE) & $18(33.9)$ & $9(17.6)$ & 0.33 \\
\hline Tiempo de hemodiálisis (DE) & $23(43.3)$ & $2(3.9)$ & $15(29.4)$ \\
\hline Tiempo de dx de IRC (DE) & $2(3.7)$ & $15(29.4)$ & 0.09 \\
\hline
\end{tabular}

DE: desviación estándar; IVU: infección de las vías urinarias; dx: diagnóstico; IRC: insuficiencia renal crónica.

Tabla 2. Características quirúrgicas de las fístulas

\begin{tabular}{|c|c|c|c|}
\hline Características & $\begin{array}{l}\text { Grupo clínico } \\
(n=53)\end{array}$ & $\begin{array}{l}\text { Grupo } \\
\text { ultrasonido } \\
(\mathbf{n}=51)\end{array}$ & $\mathbf{p}$ \\
\hline $\begin{array}{l}\text { Tipo de fístula (\%) } \\
\text { Radiocefálica distal } \\
\text { Braquicefálica } \\
\text { Braquimediana } \\
\text { Braquibasílica } \\
\text { Braquicomunicante } \\
\text { posterior }\end{array}$ & $\begin{array}{c}8(15) \\
24(45.2) \\
10(18.8) \\
9(16.9) \\
2(3.7)\end{array}$ & $\begin{array}{l}3(5.8) \\
25(49) \\
13(25) \\
5(9.8) \\
5(9.8)\end{array}$ & 0.23 \\
\hline $\begin{array}{l}\text { Tipo de anastomosis } \\
(\%) \\
\text { Término-lateral } \\
\text { Término-terminal }\end{array}$ & $\begin{array}{c}50(94.3) \\
3(5.6)\end{array}$ & $\begin{array}{c}45 \text { (88.2) } \\
6(11.7)\end{array}$ & 0.26 \\
\hline Thrill inmediato (\%) & $48(90)$ & $46(90.1)$ & 0.94 \\
\hline Maduración (\%) & $35(66)$ & $35(68.6)$ & 0.77 \\
\hline
\end{tabular}

puede ayudar a los cirujanos vasculares a planificar la mejor ubicación de la fístula, seleccionando los mejores vasos sanguíneos para crearla. La proporción de fístulas que maduran es importante debido a las complicaciones que pueden existir, como son la colocación de catéteres venosos centrales incrementado el riesgo de estenosis venosa central e infección. La obstrucción o falta de maduración de los vasos representa del 20 al $54 \%$ de los casos de falla primaria de la FAV. En nuestro estudio no se encontraron diferencias
Tabla 3. Factores de riesgo para alcanzar maduración

\begin{tabular}{|l|c|c|c|}
\hline Factor de riesgo & HR & IC & p \\
\hline Sexo & 0.7 & $0.46-1.31$ & 0.35 \\
\hline Edad & 1 & $0.9-1.02$ & 0.45 \\
\hline Hipertensión & 1.3 & $0.5-2.8$ & 1.31 \\
\hline Diabetes & 1.1 & $0.6-1.7$ & 0.6 \\
\hline Causa de IRC no & 1.1 & $0.9-1.1$ & 0.7 \\
\hline N. ${ }^{0}$ de catéteres no & 0.9 & $0.8-1.07$ & 0.34 \\
\hline Ant. diálisis peritoneal no & 1.5 & $0.9-2.6$ & 0.08 \\
\hline Tiempo de hemodiálisis & 0.9 & $0.9-1$ & 0.3 \\
\hline Tiempo de IRC & 0.9 & $0.9-1$ & 0.5 \\
\hline Tipo de fístula & 1 & $0.8-1.1$ & 0.7 \\
\hline Tipo de anastomosis & 0.8 & $0.3-2.2$ & 0.7 \\
\hline Thrill & 1.5 & $0.5-4.1$ & 0.4 \\
\hline Lugar de la fístula & & & \\
\hline
\end{tabular}

HR: hazard ratio; IC: intervalo de confianza; IRC: insuficiencia renal crónica.

estadísticamente significativas con respecto a la falta de maduración y la edad del paciente. Esto va de acuerdo con la literatura, en donde se reportan estudios donde se concluye que no existe una asociación clara entre la falta de maduración y la edad. Así mismo, no se encontró una relación entre el sexo y la maduración, 
Tabla 4. Análisis multivariante por factores de riesgo

\begin{tabular}{|l|c|c|c|}
\hline Factor de riesgo & HR & IC & p \\
\hline Sexo & 1 & $0.98-1.02$ & 0.54 \\
\hline Edad en años & 0.7 & $0.45-1.32$ & 0.35 \\
\hline Hipertensión & 1.1 & $0.47-2.6$ & 0.78 \\
\hline Diabetes & 1.1 & $0.58-1.75$ & 0.95 \\
\hline
\end{tabular}

Tabla 5. Análisis multivariante por tipo de fístula

\begin{tabular}{|l|c|c|c|}
\hline Factor de riesgo & HR & IC & p \\
\hline Tipo de fístula & & & \\
\hline Radiocefálica distal & 0.34 & $0.003-0.36$ & 0.005 \\
Braquicefálica & 0.27 & $0.003-2.62$ & 0.002 \\
Braquibasílica & 0.40 & $0.004-0.425$ & 0.008 \\
Braquimediana & 0.33 & $0.003-0.334$ & 0.004 \\
Braquicomunicante posterior & 0.25 & $0.002-0.285$ & 0.003 \\
\hline
\end{tabular}

HR: hazard ratio; IC: intervalo de confianza.

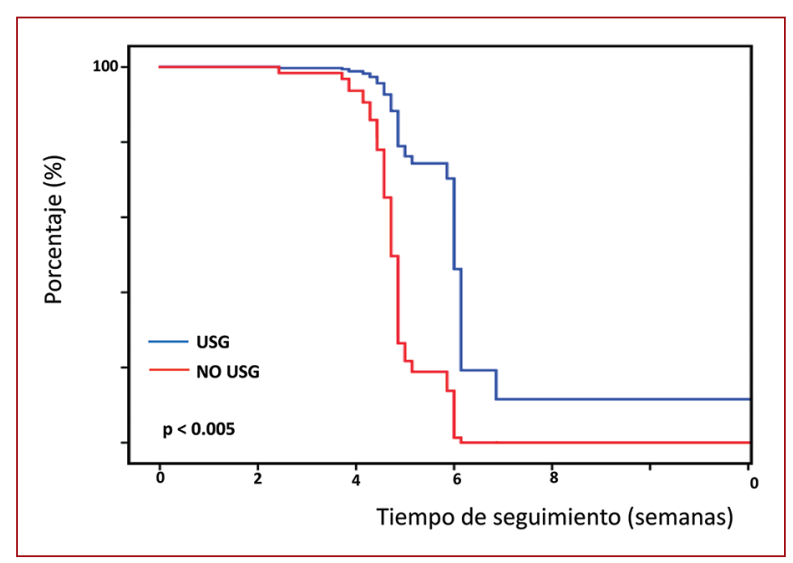

Figura 1. Curva de supervivencia de maduración clínica. USG: ultrasonido.

como se reporta en artículos de revisión donde no hay diferencia entre ambos grupos. Con respecto a la hipertensión y a la diabetes, en nuestro estudio no hubo diferencia entre el éxito de maduración y la asociación con estas enfermedades. Se reporta que la diabetes influye en la remodelación de los vasos, pero no se encontró una asociación clara; lo mismo ocurre con la hipertensión, en la cual no se observan diferencias entre presentarla y mejorar el pronóstico de maduración.

Por lo anterior, en este estudio se evaluaron los factores de riesgo para maduración, así como comparar el tiempo de esta en un grupo de pacientes a los cuales se les realizó mapeo preoperatorio frente a aquellos a los que no se les realizó. Se analizaron un total de 104 pacientes de los cuales a 51 se les realizó mapeo preoperatorio y a 53 se les realizó la cirugía solo con EP. En un estudio de Cochrane de 2015 no apoyan la evidencia de realizar USG preoperatorio debido a que este no mostraba ser superior para la maduración con respecto a los pacientes a los que no se les realizó USG ${ }^{8}$.

De los pacientes que se analizaron, la principal causa de enfermedad renal en ambos grupos fue diabetes, siendo en el GC 24 (45.2) y en el grupo de USG 27 (52.9). Con respecto a las características quirúrgicas, el procedimiento que más se realizó fue la fístula braquicefálica siendo de 25 (49) en el grupo USG y de 24 (45.2) en el GC. Al hacer el análisis estadístico entre ambos grupos, no hubo diferencias significativas con respecto a la maduración y realizar el mapeo preoperatorio, encontrando que en ambos grupos se alcanzó el mismo porcentaje de maduración, sin embargo, en el grupo en el cual se realizó el rastreo ultrasonográfico estos alcanzaron una maduración en menos tiempo de seguimiento, con una diferencia estadísticamente significativa ( $p=0.001$; HR: 0.1 ; IC 95\%: 0.08-0.3). El rastreo ultrasonográfico previo a la construcción de una FAV ayuda a la identificación de alteraciones que no son evidentes a la EP. Por lo tanto, ayuda a una mejor planeación con respecto a la localización. Nuestro estudio muestra que el mapeo preoperatorio sistemático, además de la $\mathrm{EP}$, mejora el tiempo de maduración de las fístulas en una población de pacientes sin problemas de acceso complejos. Sin embargo, no se observaron diferencias significativas entre ambos grupos, ya que del grupo al que se le realizó USG maduraron 35 (33.7) y en el grupo al que solo se les realizó EP maduraron 35 (33.7). Para poder lograr diferencias estadísticamente significativas se debe aumentar el tamaño de la muestra. Así mismo, al hacer el análisis multivariante con respecto a la localización se encontró que sí hubo diferencias estadísticamente significativas en las fístulas braquicefálicas ( $p=0.002$; HR: 0.27; IC 95\%: 0.003-2.62), así como en las fístulas braquicomunicantes posteriores $(p=$ 0.003; HR: 0.25; IC 95\%: 0.002-0.285) con respecto a la maduración.

\section{Conclusiones}

Se debe realizar mapeo ultrasonográfico como herramienta preoperatoria en la planeación de la construcción de fístulas arteriovenosas para HD. Se observó 
que el realizar USG preoperatorio en los pacientes del Hospital General de México que serán sometidos a construcción de FAV reduce el tiempo de maduración de esta. No se observaron diferencias estadísticamente significativas con respecto a la maduración entre ambos grupos. Para lograrlas se deben ampliar el tamaño de la muestra y el tiempo de seguimiento. Estas fueron las principales limitantes de nuestros estudio, contar con una cantidad pequeña de pacientes en un periodo corto de tiempo. EI USG preoperatorio demostró ser una herramienta indispensable, ya que permite realizar una mejor planeación y mejorar los resultados en las fístulas arteriovenosas.

\section{Declaraciones éticas}

Este estudio no contempló la intervención o la manipulación de tratamiento de los pacientes del servicio de angiología y cirugía vascular a quienes se les realizó FAV. Únicamente contempló el análisis de los registros ya existentes. El estudio se llevó a cabo de conformidad con el Reglamento de la Ley General de Salud en Materia de Investigación en Salud e ICH-GCP, con apego a la ética y salvaguarda de la privacidad y confidencialidad de la información obtenida, además de que la información se utilizará exclusivamente para fines académicos y de investigación.

\section{Agradecimientos}

Se agradece a los médicos de base que ayudaron a la realización de este estudio, así como a los médicos residentes de angiología del Hospital General de México "Dr. Eduardo Liceaga" por el apoyo brindado durante la realización de este proyecto.

\section{Financiamiento}

La presente investigación no ha recibido ninguna beca específica de agencias de los sectores públicos, comercial o sin ánimo de lucro.

\section{Conflicto de intereses}

Los autores declaran no tener conflicto de intereses.

\section{Responsabilidades éticas}

Protección de personas y animales. Los autores declaran que para esta investigación no se han realizado experimentos en seres humanos ni en animales.

Confidencialidad de los datos. Los autores declaran que han seguido los protocolos de su centro de trabajo sobre la publicación de datos de pacientes.

Derecho a la privacidad y consentimiento informado. Los autores han obtenido el consentimiento informado de los pacientes y/o sujetos referidos en el artículo.

\section{Bibliografía}

1. Webster A, Nagler E, Morton R, Masson P. Chronic kidney disease. Lancet. 2017;389(10075):1238-52.

2. Bashar K, Conlon P, Kheirelseid E, Aherne T, Walsh S, Leahy A. Arteriovenous fistula in dialysis patients: Factors implicated in early and late AVF maturation failure. Surgeon. 2016;14(5):294-300.

3. Asif A, Roy-Chaudhury P, Beathard GA. Early arteriovenous fistula failure: a logical proposal for when and how to intervene. Clin J Am Soc Nephrol. 2006;1(2):332-9.

2. Itoga N, Ullery B, Tran K, Lee G, Aalami O, Bech F, et al. Use of a proactive duplex ultrasound protocol for hemodialysis access. J Vasc Surg. 2016;64(4):1042-9.

5. Ernandez T, Saudan P, Berney T, Merminod T, Bednarkiewicz M, Martin P-Y. Risk factors for early failure of native arteriovenous fistulas. Nephron Clin Pract. 2005;101(1):c39-44.

6. Marques M, Ponce P. Pre-operative assessment for arteriovenous fistula placement for dialysis. Semin Dial. 2017;30(1):58-62 